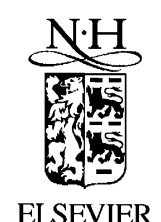

Nuclear Instruments and Methods in Physics Research A 478 (2002) 357-361

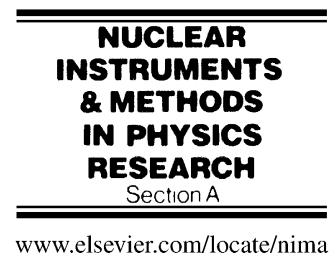

\title{
CCD readout of GEM-based neutron detectors
}

\author{
F.A.F. Fraga ${ }^{a}$ *, L.M.S. Margato ${ }^{a}$, S.T.G. Fetal ${ }^{a}$, M.M.F.R. Fraga ${ }^{a}$, \\ R. Ferreira Marques ${ }^{\mathrm{a}}$, A.J.P.L Policarpo ${ }^{\mathrm{a}}$, B. Guerard ${ }^{\mathrm{b}}$, A. Oed ${ }^{\mathrm{b}}$, \\ G. Manzini ${ }^{\mathrm{b}}, \mathrm{T}$. van Vuure ${ }^{\mathrm{c}}$ \\ ${ }^{a}$ LIP-Coimbra and Departamento de Física da Universidade de Coimbra, 3004-516 Coimbra, Portugal \\ ${ }^{\mathrm{b}}$ Institute Laue Langevin, BP $156 X$, F-38042 Grenoble Cedex, France \\ ${ }^{\mathrm{c}}$ Delft University of Technology, IRI-ISO, Mekelweg 15, NL $2629 \mathrm{JB}$ Delft, The Netherlands
}

\begin{abstract}
We report on the optical readout of the gas electron multiplier (GEM) operated with a gaseous mixture suitable for the detection of thermal neutrons: ${ }^{3} \mathrm{He}-\mathrm{CF}_{4}$. A CCD system operating in the $400-1000 \mathrm{~nm}$ band was used to collect the light. Spectroscopic data on the visible and NIR scintillation of $\mathrm{He}-\mathrm{CF}_{4}$ are presented. Images of the tracks of the proton and triton recorded with a triple GEM detector are also shown. (C) 2002 Elsevier Science B.V. All rights reserved.
\end{abstract}

PACS: $29.40 . \mathrm{Gx}$

Keywords: Gas electron multiplier; Neutron detector; Optical readout

\section{Introduction}

Gas detectors filled with ${ }^{3} \mathrm{He}$ are commonly used for the detection of thermal neutrons mainly because of their high efficiency and low gamma sensitivity. The use of the scintillation emitted by the electron avalanches developed in the microstructures has been reported before [1]. We have also presented some previous work on the use of the scintillation of the gas electron multiplier (GEM) avalanches for imaging purposes, of both $\mathrm{X}$-rays and charged particles, using a high-resolution cooled CCD camera [2]. In the previous work, we anticipated that using appropriate gaseous

\footnotetext{
*Corresponding author. LIP-Coimbra, Departamento de Fisica, Universidade de Coimbra, 3000 Coimbra, Portugal. Tel.: +351-39-833-465; fax: +351-39-822-358.

E-mail address: francisco@lipc.fis.uc.pt (F.A.F. Fraga).
}

mixtures and several cascaded GEMs, CCDs could be used to image tracks produced by the products resulting from the interaction between thermal neutrons and the ${ }^{3} \mathrm{He}$ atom.

In this paper, we present data concerning the use of $\mathrm{He}-\mathrm{CF}_{4}$ gaseous mixtures in GEM detectors, a description of a detector that we built to image the tracks of the proton and triton and some sample images obtained with this set-up.

\section{The experimental set-up}

The characteristics of the GEMs currently available were optimized for operation at typical LHC applications using the deeply studied $\mathrm{Ar}-$ DME or Ar- $\mathrm{CO}_{2}$ mixtures [3] and the operation of GEMs with $\mathrm{CF}_{4}$ mixtures needs higher-magnitude 
electrical fields, as presented in Ref. [2]. Taking into account that we also intended to perform measurements at high pressure, we had some GEMs with double conical holes manufactured ${ }^{1}$ with hole dimensions narrower than usual. In this work, we used $5 \times 5 \mathrm{~cm}^{2}$ GEMs having 45, 60 and $80 \mu \mathrm{m}$ (standard GEM) metal diameter holes with 35,50 and $70 \mu \mathrm{m}$ kapton holes, respectively. The kapton thickness was $50 \mu \mathrm{m}$ and pitch was $140 \mu \mathrm{m}$ for all GEMs. Considering the high price of ${ }^{3} \mathrm{He}$, all the preliminary charge and scintillation measurements were taken using high-purity ${ }^{4} \mathrm{He}$ using an $\mathrm{X}$-ray tube, as no significant difference is expected in the charge and scintillation properties of these two isotopes when mixed with $\mathrm{CF}_{4}$.

As the experimental system and detector that were used to obtain these preliminary results are similar to the ones described in Ref. [2], we will only refer to the more relevant aspects. The GEM front electrode was grounded, the back one was operated at negative voltage. A drift plane was placed $6 \mathrm{~mm}$ before the GEM, defining a collection zone. Although an optically transparent collecting grid was placed $2 \mathrm{~mm}$ far from the GEM, in these experiments, it was kept at the same voltage as the outer GEM electrode and all the avalanche electrons were collected by the GEM electrode. The light window, placed behind the GEM, was made of $6 \mathrm{~mm}$ thick glass with a $5 \mathrm{~cm}$ effective diameter. The ratio between the secondary and the primary currents, measured at the GEM back electrode and when operating the chamber in ionization mode, respectively, were used to calculate the GEM gains.

A Quantix 1400 camera, manufactured by Photometrics Ltd., was used to read out the light emitted from the GEM. It uses a Peltier cooled, low-noise CCD, KAF 1400 from Kodak, with $1317 \times 1035$ pixels having a size of $6.8 \times 6.8 \mu \mathrm{m}^{2}$. The spectral response goes from 400 to $1000 \mathrm{~nm}$. All the pictures presented in this work were taken with the CCD cooled to $-30^{\circ} \mathrm{C}$. A standard $50 \mathrm{~mm}$ $\mathrm{f}: 1.8$ photographic lens was used and the camera

\footnotetext{
${ }^{1}$ CERN Surface Treatment group.
}

was placed at the minimum allowable focusing distance, about $30 \mathrm{~cm}$ away from the chamber glass window.

\section{Scintillation and charge data}

The data on charge gain in $\mathrm{He}(600 \mathrm{mbar})+\mathrm{CF}_{4}$ (400 mbar) are shown in Fig. 1. The drift field was carefully adjusted for maximum GEM transparency. Gain with pure $\mathrm{CF}_{4}$ at $400 \mathrm{mbar}$ was also measured and, as expected, the addition of 0.6 bar of $\mathrm{He}$ has a negligible effect compared to the data represented, independent of the hole diameter. However, it was seen that the addition of 3.6 bar of

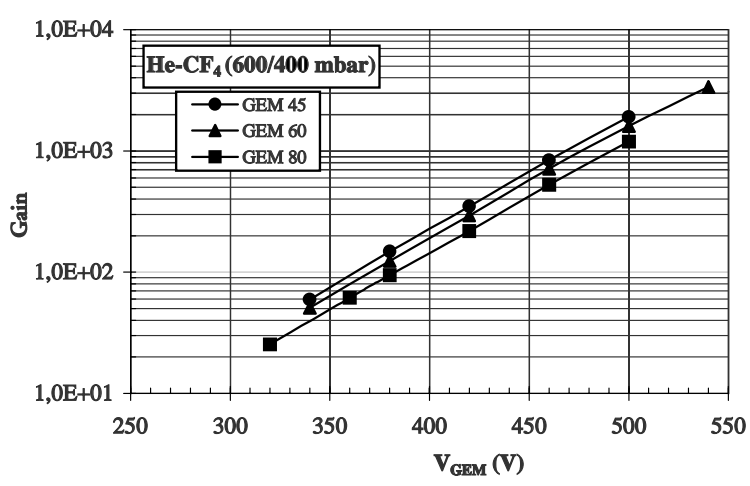

Fig. 1. GEM gains measured in $\mathrm{He}(600 \mathrm{mbar})-\mathrm{CF}_{4}$ (400 mbar) as a function of $V_{\mathrm{GEM}}$ for GEMs with 45,60 and $80 \mu \mathrm{m}$ hole diameter.

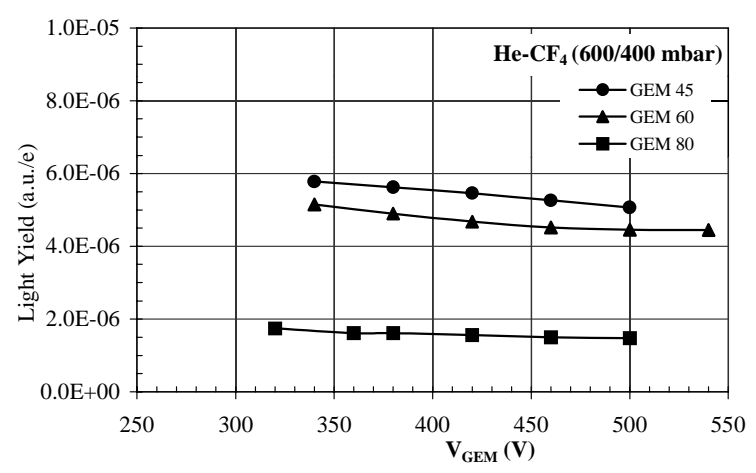

Fig. 2. Ratio of emitted light over secondary electron current versus $V_{\mathrm{GEM}}$ measured in $\mathrm{He}(600 \mathrm{mbar})+\mathrm{CF}_{4}$ ( $\left.400 \mathrm{mbar}\right)$ for GEMs with 45, 60 and $80 \mu \mathrm{m}$ hole diameter. 
He shifted the gain curve towards higher voltage of about $30 \mathrm{~V}$ for the 60 and $45 \mu \mathrm{m}$ holes, and about $70 \mathrm{~V}$ for the $80 \mu \mathrm{m}$ ones. Measurements taken on

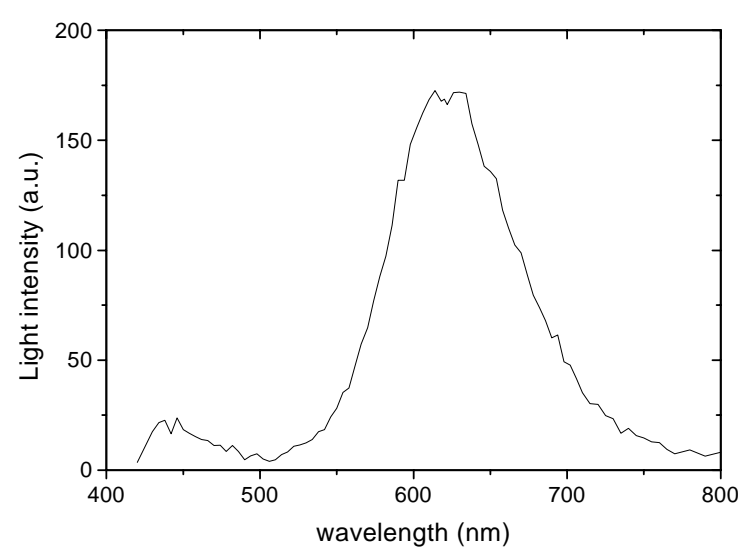

Fig. 3. Emission spectrum in $\mathrm{He}(600 \mathrm{mbar})+\mathrm{CF}_{4}(400 \mathrm{mbar})$ gas mixture measured at charge gain of $190(\Delta \lambda=5 \mathrm{~nm})$.

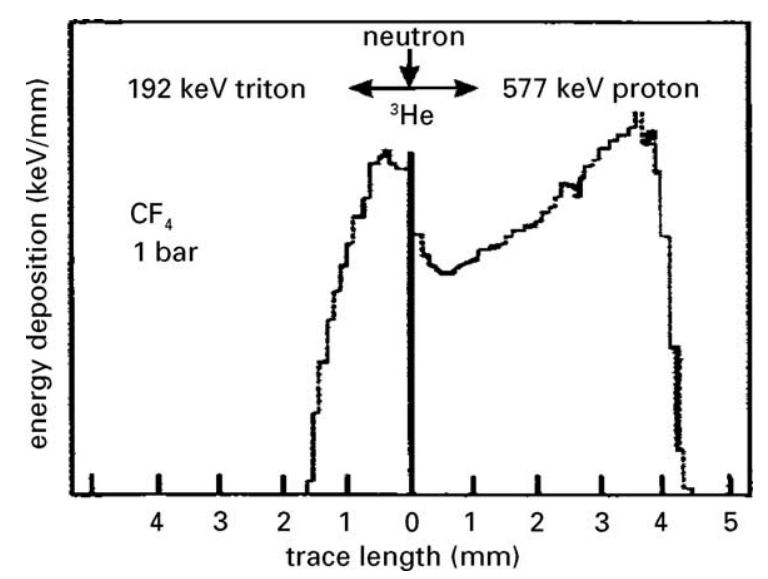

Fig. 4. The energy deposition along the proton and triton track in 1 bar $\mathrm{CF}_{4}$. the collection and on the transparency of these GEMs also suggested that the pitch of the GEMs should be changed in future designs to improve electron transfer between GEMs.

Fig. 2 shows the data on scintillation measured in the same conditions as in the previous figure. The GEMs with $45 \mu \mathrm{m}$ holes have higher light emission than the other GEMs. The measured emission spectrum of the $\mathrm{He}-\mathrm{CF}_{4}$ mixture is shown in Fig. 3. The CCD has a mean quantum efficiency of $35 \%$ at the light peak.

The ${ }^{3} \mathrm{He}-\mathrm{CF}_{4}$ mixture is commonly used in gaseous neutron detectors. The ${ }^{3} \mathrm{He}$ is used for neutron conversion $\left({ }^{3} \mathrm{He}+\mathrm{n} \Rightarrow \mathrm{p}+{ }^{3} \mathrm{H}+764 \mathrm{keV}\right)$ and the $\mathrm{CF}_{4}$ is needed to decrease the range of the proton and the triton. Fig. 4 shows the energy deposition along the straight track in 1 bar $\mathrm{CF}_{4}$ [4]. The centroid of the distribution has an offset of approximately $40 \%$ of the proton track, limiting the position resolution of this type of detectors. As our purpose was to obtain images of tracks about $1 \mathrm{~cm}$ long, we decided to operate the detector with a $\mathrm{CF}_{4}$ partial pressure of 400 mbar.

For the neutron measurements, the radiation entrance of the detector was replaced with a $6 \mathrm{~mm}$ thick aluminium window. Fig. 5 shows a diagram of the detector, a cascaded triple GEM was used. GEMs with $80 \mu \mathrm{m}$ holes were used for the entrance and intermediate positions and a $60 \mu \mathrm{m}$ one was used as outer GEM. The entrance window was grounded and the absorption zone was $6 \mathrm{~mm}$ deep. The distance between the GEMs was $2 \mathrm{~mm}$.

An AmBe neutron source with polyethylene shielding was used for the data taking, carried out at the ILL Grenoble. Fig. 6 shows some images of

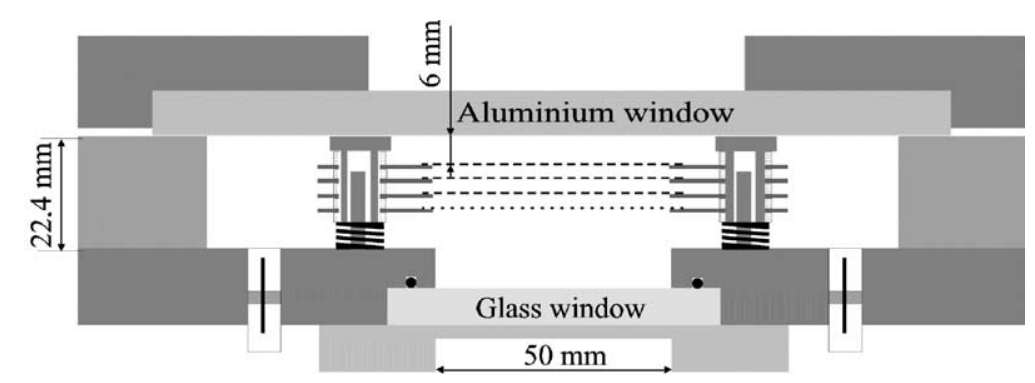

Fig. 5. Schematic cross-section of the detector. The CCD (not shown) was placed $30 \mathrm{~cm}$ away from the glass window. 


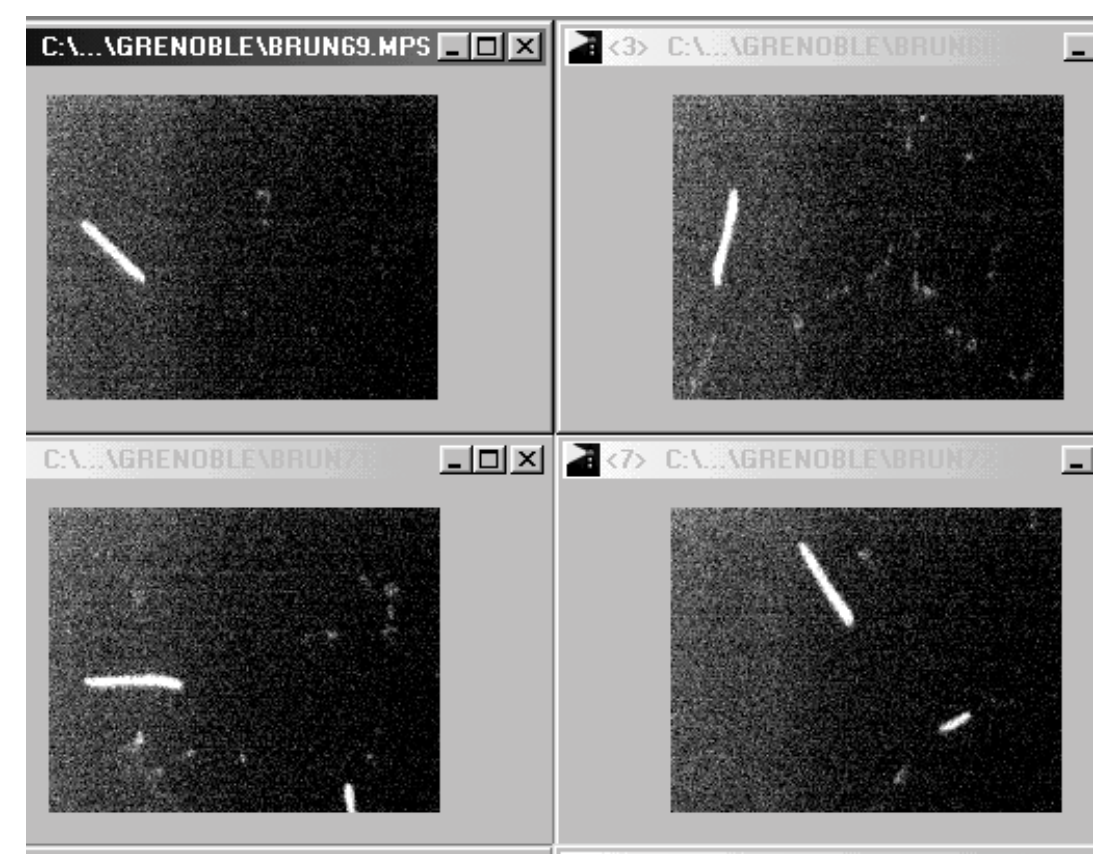

Fig. 6. Images of proton and triton tracks obtained with $\mathrm{He}(1 \mathrm{bar})-\mathrm{CF}_{4}(400 \mathrm{mbar}): V_{\mathrm{GEM} 1}=V_{\mathrm{GEM} 2}=V_{\mathrm{GEM} 3}=400 \mathrm{~V}, E_{\mathrm{D}}=1 \mathrm{kV} /$ $\mathrm{cm}, E_{\mathrm{T}}=3 \mathrm{kV} / \mathrm{cm}, \mathrm{CCD}$ binning $7 \times 7, T_{\exp }=10 \mathrm{~ms}$.

the projections of the proton and triton tracks, taken with the CCD camera using a $7 \times 7$ binning and an exposition time of $10 \mathrm{~ms}$ with a 1 bar ${ }^{3} \mathrm{He}-$ $400 \mathrm{mbar} \mathrm{CF}_{4}$ filling. The co-linear structure of the tracks is clearly seen in most events, although a few angled tracks, corresponding to higher energy neutrons were also recorded. The track with the shorter length, shown in the bottom-right CCD image corresponds to a more inclined track or to wall effects.

Fig. 7 shows, in more detail, two images of tracks and the respective distribution of the scintillation light along the longitudinal CCD pixels of the track projection. The light variation along the track, due to the different energy deposition curves of the proton and the triton, are easily identified and should be compared with Fig. 4. Some superimposed tracks, obtained with a longer exposition time (1 s) and slightly higher $\mathrm{CF}_{4}$ partial pressure, implying shorter tracks, are shown in Fig. 8. Some interactions due to the gamma background are also seen.

\section{Conclusions}

A detector with a cascaded triple GEM has been successfully operated with $\mathrm{He}-\mathrm{CF}_{4}$ mixtures and the optical readout of ${ }^{3} \mathrm{He}$ neutron GEM detectors has been done using a simple CCD system. The information about charge deposits along the tracks can be easily extracted from the images and could be useful for avoiding wall-effect errors in energy measurements, or for neutron spectroscopy purposes that rely on track angle [5].

The GEM geometry should be optimized for scintillation. Thicker GEMs should have higher light yields, and the performance of our system is being improved using GEMs with narrow holes of smaller pitch.

Applications of the scintillation light emitted by the GEM, using photon-counting readouts, are also being considered and data on the operation of GEMs with $\mathrm{He}-\mathrm{CF}_{4}$ mixtures at higher pressures will also be reported soon. 

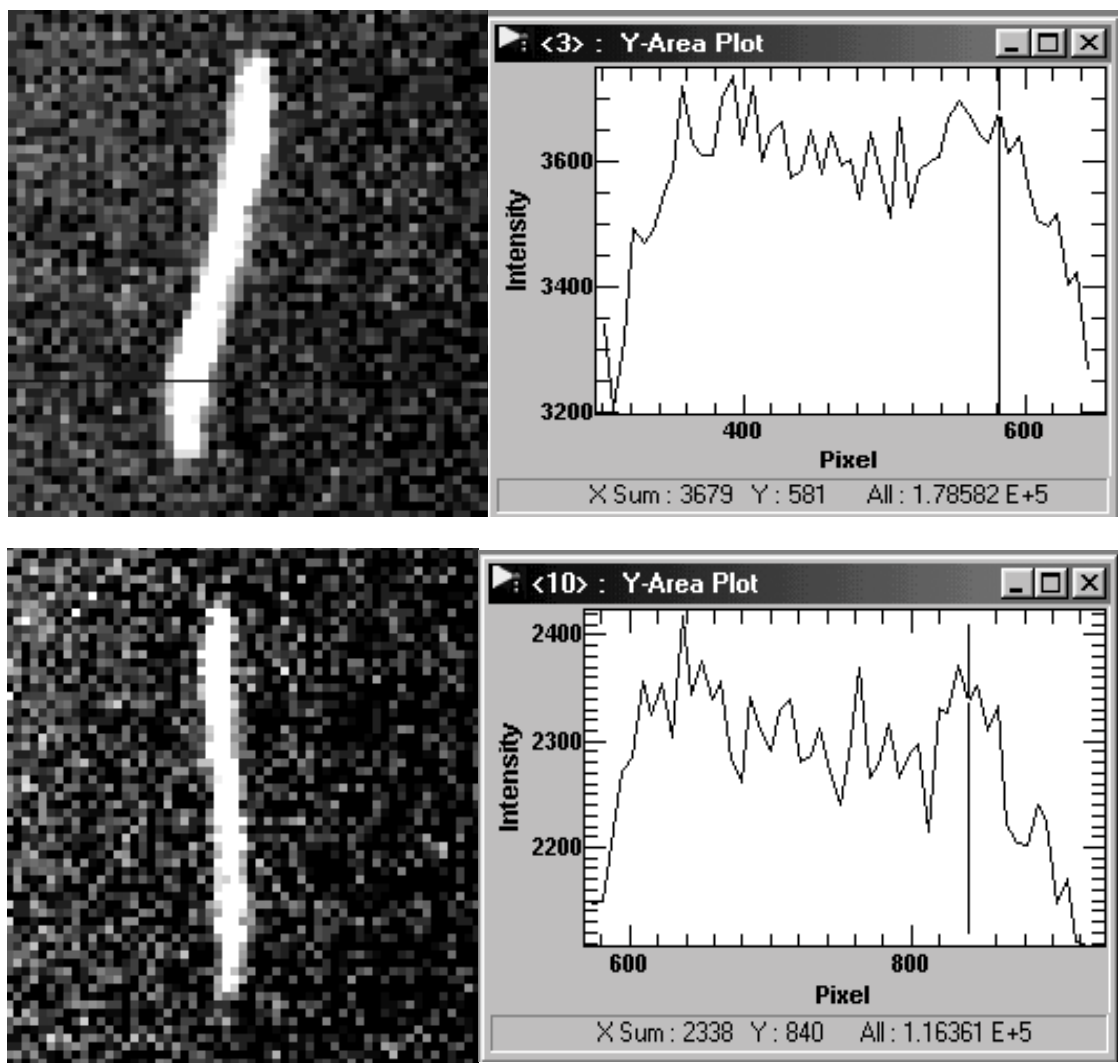

Fig. 7. Distribution of measured scintillation along tracks. The Bragg curves of the proton (left) and triton (right) are revealed.

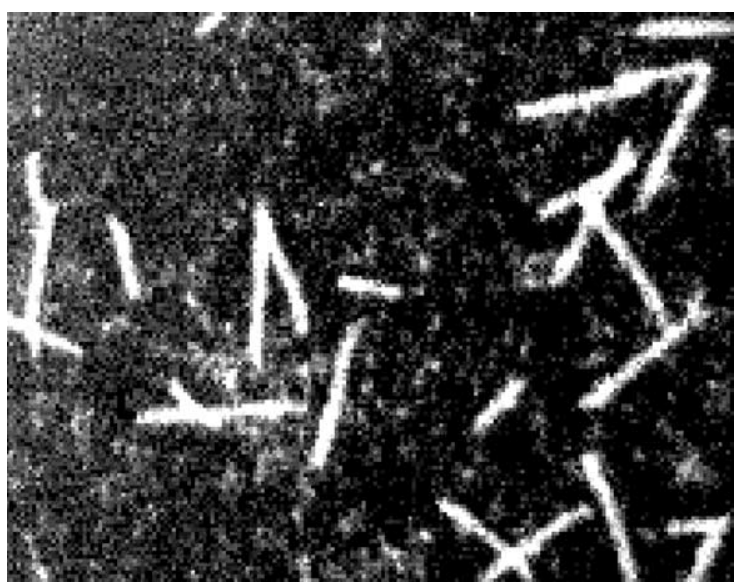

Fig. 8. Superimposed proton-triton tracks obtained with an exposition time of $1 \mathrm{~s}$.

\section{Acknowledgements}

This work was supported by the contract CERN/P/FIS/15199/99 with the Portuguese FCT. Thanks are due to F. Sauli who made available some of the GEM foils used in this work.

\section{References}

[1] F.A.F. Fraga, et al., Nucl. Instr. and Meth, A471 (2001) 125.

[2] F.A.F. Fraga, L.M.S. Margato, S.T.G. Fetal, R. Ferreira Marques, A.J.P.L Policarpo, Presented at the Nuclear Science Symposium and Medical Imaging Conference, Lyon, France, 15-20 October, 2000, Trans. Nucl. Sci., accepted for publication.

[3] J. Benlloch, et al., Nucl. Instr. and Meth. A 419 (1998) 410.

[4] F.D. van den Berg, Ph.D Thesis, TUDelft, 2000.

[5] U. Titt, et al., Nucl. Instr. and Meth. A 416 (1998) 85. 